\title{
Article
}

\section{On Markov Moment Problem and Related Results}

\author{
Octav Olteanu
}

check for updates

Citation: Olteanu, O. On Markov Moment Problem and Related Results. Symmetry 2021, 13, 986. https://doi.org/10.3390/ sym13060986

Academic Editor: Alina Alb Lupas

Received: 22 April 2021

Accepted: 25 May 2021

Published: 1 June 2021

Publisher's Note: MDPI stays neutral with regard to jurisdictional claims in published maps and institutional affiliations.

\section{Copyright: (C) 2021 by the author.} Licensee MDPI, Basel, Switzerland. This article is an open access article distributed under the terms and conditions of the Creative Commons Attribution (CC BY) license (https:// creativecommons.org/licenses/by/ $4.0 /)$.
Department of Mathematics-Informatics, University Politehnica of Bucharest, Splaiul Independenţei 313, 060042 Bucharest, Romania; octav.olteanu50@gmail.com; Tel.: +40-728-301-872

\begin{abstract}
We prove new results and complete our recently published theorems on the vector-valued Markov moment problem, by means of polynomial approximation on unbounded subsets, also applying an extension of the positive linear operators' result. The domain is the Banach lattice of continuous real-valued functions on a compact subset or an $L_{v}^{1}$ space, where $v$ is a positive moment determinate measure on a closed unbounded set. The existence and uniqueness of the operator solution are proved. Our solutions satisfy the interpolation moment conditions and are between two given linear operators on the positive cone of the domain space. The norm controlling of the solution is emphasized. The most part of the results are stated and proved in terms of quadratic forms. This type of result represents the first aim of the paper. Secondly, we construct a polynomial solution for a truncated multidimensional moment problem.
\end{abstract}

Keywords: polynomial approximation; constrained extension of linear operators; Markov moment problem; self-adjoint operator; symmetric matrix; compact subset; spectrum; unbounded subset; basic nonnegative polynomials; truncated moment problem

\section{Introduction}

The one dimensional classical moment can be formulated as follows: for a given sequence $\left(y_{n}\right)_{n \geq 0}$ of real numbers, find a positive measure $v$ on the given interval $\mathbb{I}$ (the important cases being $\mathbb{I}=\mathbb{R}, \mathbb{I}=[0, \infty)$ or $\mathbb{I}=[0,1]$ ), such that the following applies:

$$
\int_{\mathbb{I}} t^{n} d v=y_{n}, n=0,1,2, \ldots
$$

where the numbers $y_{n}, n=0,1,2, \ldots$ are called the moments of the measure $d v$ (or $v$ ) on the interval $\mathbb{I}$. In the sequel, the notations are as follows:

$$
\mathbb{N}=\{0,1,2, \ldots\}, \mathbb{R}_{+}=[0, \infty), p r_{j}: \mathbb{R}^{n} \rightarrow \mathbb{R}, p r_{j}\left(t_{1}, \ldots, t_{j}, \ldots, t_{n}\right)=t_{j}, j=1, \ldots, n, n \geq 2 .
$$

If the intervals $\mathbb{I}_{j} \subseteq \mathbb{R}$, and $f_{j}: \mathbb{I}_{j} \rightarrow \mathbb{R}, j=1, \ldots, n$ are given, we denote by the following:

$$
f_{1} \otimes \cdots \otimes f_{n}: \mathbb{I}_{1} \times \cdots \times \mathbb{I}_{n} \rightarrow \mathbb{R}
$$

The function is defined as follows: $\left(f_{1} \otimes \cdots \otimes f_{n}\right)\left(t_{1}, \ldots, t_{n}\right)=f_{1}\left(t_{1}\right) \cdots f_{n}\left(t_{n}\right)$. When the interval appearing in (1) is $\mathbb{I}=\mathbb{R}$, the moment problem is a Hamburger moment problem. If $\mathbb{I}=\mathbb{R}_{+}$, then the corresponding problem is a Stieltjes moment problem, while in the case $\mathbb{I}=[0,1]$ we have a Hausdorff moment problem. The classical moment problem makes sense in the multidimensional case, when $\mathbb{I}$ appearing in (1) is replaced by an arbitrary closed subset $S$ of $\mathbb{R}^{n}, n \geq 2, t^{j}=t_{1}^{j_{1}} \cdots t_{n}^{j_{n}}, t=\left(t_{1}, \ldots, t_{n}\right) \in S, j=$ $\left(j_{1}, \ldots, j_{n}\right) \in \mathbb{N}^{n}$. One denotes by $C_{0}(S)$, the space of all continuous real-valued compactly supported functions defined on $S$. An important case is when the solution is a positive linear functional or operator defined on $L_{v}^{1}(S)$. In this case, $v$ is assumed to be a positive 
regular Borel measure on $S$. A basic particular case is that of a determinate measure $v$, with finite moments of all orders:

$$
\int_{S} t^{j} d v \in \mathbb{R}, j \in \mathbb{N}^{n} .
$$

We recall that the measure $v$ is called a moment determinate (or determinate) if it is uniquely determined by its moments, or, equivalently, by its values on the subspace $\mathcal{P}$ of all polynomials. By $\mathcal{P}_{+}=\mathcal{P}_{+}(S)$, we denote the convex cone of all polynomial functions that are nonnegative on $S$. The problem may be called an $n$-dimensional Hamburger, Stieltjes or Hausdorff moment problem, depending on the cases $S=\mathbb{R}^{n}, S=\mathbb{R}_{+}^{n}$ or $S=[0,1]^{n}$. By its formulation, the moment problem is an inverse problem, since the measure $v$ is not known. The words "find a positive measure $v$ " refer to characterizing the existence of $v$ in terms of the given moments $y_{n}, n \geq 0$ such that (1) holds, to establish the uniqueness (or non-uniqueness) of the (or a) solution $v$, and, eventually, to its construction. The direct problem would be as follows: given the measure $v$, find its moments defined by (1). This seems to be a computational problem. Going back to the moment problem, if an upper bound is imposed on $v$, we have a Markov moment problem, which is closely related to the $L$-moment problem. In its general formulation, the classical multidimensional real moment problem is similar to that mentioned above, when the interval $\mathbb{I}$ is replaced by a closed (bounded or unbounded) subset $S \subseteq \mathbb{R}^{n}, n \geq 2$. For the classical moment problem, including modern approaches of it, see the monographs [1-3], and most of the articles on the moment problem listed in the references. For the background, ordered topological vector spaces, linear operators, and the recent results in analysis and functional analysis see [4-9]. Earlier and recent results on the classical moment problem can be found in [10-18]. The papers $[19,20]$ deal with the basic properties of convex operators in terms of the associated linear operators. The proofs are partially based on Hahn-Banach type theorems. The article [21] presents the density theorem and a Bishop type theorem in a special set of continuous functions, while the references [22-27] concern results on the classical moment problem and aspects of its connections with other fields of functional analysis. Finally, articles [28-38] refer to the Markov moment problem and the related areas of research. In solving Markov moment problems, there are three classes of methods recalled below, which are combined to reach the existence, uniqueness and construction of the solutions for the problems under discussion in the present paper. The interested reader can find additional information by means of the references here and from other sources. Previous similar main results on the construction of solutions for reduced moment problems were published in [28,30,31] and in Section 4 of [35]. When the solution is not a polynomial, finding it leads to a system of nonlinear equations. For example, in [31], a step-function solution $f$ is under attention. Namely, finding $f$ of [31] leads to a system of nonliear equations. In turn, such a solution $f$ verifies $0 \leq f \leq 1$; this is not the case for our solution $h$ given by (7) and (8). Namely, the evaluations (12) and (13) proved in Section 3.2 of the present paper ensure neither $h \geq 0$ nor $h \leq 1$ on $S$. A quite similar problem is discussed in [30], where the resolution of the finite Markov moment problem is done through the theory of Toeplitz matrixes, also using Newton's relations for polynomials. The references deal with various aspects of the classical moment problem and its relationships with other fields (fixed point theorems, operator theory, expressing nonnegative polynomials on special closed subsets in terms of sums of squares, approximation theory, numerical analysis, algebra). In all this problems, the moment sequences given by (1) play a central role. Another formulation of the scalar valued moment problem is as follows: given a Banach function space $X$ containing all polynomials and compactly supported continuous functions on a subset $S$ of $\mathbb{R}^{n}, n \geq 2$, find a necessary and sufficient condition on the sequence of moments for the existence of a positive element $x^{\star}$ in the dual $X^{\star}$ of that Banach space of functions on a closed subset $S$, such that $x^{\star}\left(\varphi_{j}\right)=y_{j}, j \in \mathbb{N}^{n}$. Here $\varphi_{j}(t) t^{j}$, $j \in \mathbb{N}^{n}, t \in S$. In case of a Markov moment problem, we additionally require that $x^{\star}$ is dominated by a given continuous linear functional on the positive cone $X_{+}$of $X$, or $x^{\star}$ is dominated by a continuous convex functional on the whole space $X$. Adding new 
results and improving earlier information on this subject is the first aim of the present work. Here, the moments $y_{j}, j \in \mathbb{N}^{n}$ are real numbers, while in the general case (see the next sections, except Section 3.2) the moments $y_{j}$ may be vectors, functions, symmetric matrices, or self-adjoint operators. In this case, the solution is a positive linear operator $T$. A polynomial solution for the truncated moment problem, accompanied by a simple method of evaluating the norm of the vector defined by the coefficients, is also under attention. The rest of the paper is organized as follows: Section 2 summarizes theoretical more or less known methods applied in order to achieve the aims of the paper. In Section 3, we tried to achieve the goals claimed above, giving also the proofs where necessary. Section 4 discusses a part of the results, as well as possible future work on these subjects. Section 5 concludes the paper.

\section{Methods}

\subsection{Constrained Extension Results for Linear Operators}

There are two Hahn-Banach extension type theorems stated below. The first one ensures only the existence of a positive extension, while the second one additionally involves a dominating condition. In particular, this second requirement allows evaluating the norm of the solution. Such an evaluation can be achieved also by using the first extension type result (Theorem 1 stated below), then passing to the limit. Here are the main two extension results that will be applied in the sequel. Let $X_{1}$ be an ordered vector space for which the positive cone $X_{1,+}$ is generating $\left(X_{1}=X_{1,+}-X_{1,+}\right)$. Recall that in such an ordered vector space $X_{1}$, a vector subspace $\mathcal{S}$ is called a majorizing subspace if for any $x \in X_{1}$, there exists $s \in \mathcal{S}$, such that $x \leq s$. The following theorem holds true.

Theorem 1 ([19] Theorem 1.2.1). Let $X_{1}$ be an ordered vector space whose positive cone is generating, $\mathcal{S} \subset X_{1}$ a majorizing vector subspace, $Y$ an order complete vector space, $T_{0}: \mathcal{S} \rightarrow Y$ a positive linear operator. Then $T_{0}$ admits a positive linear extension $T: X_{1} \rightarrow Y$.

The next result was published in the following version in [34] Theorem 4. It can be regarded as a generalization of a result of M.G. Krein [2] to an arbitrary infinite set of moments and to vector-valued operators.

Theorem 2 ([34] Theorem 4). Let $X$ be an ordered vector space, $Y$ an order complete vector lattice, J an arbitrary set, $\left\{x_{j}\right\}_{j \in J} \subset X,\left\{y_{j}\right\}_{j \in J} \subset Y$ given families, $T_{1}, T_{2} \in L(X, Y)$ two linear operators. The following statements are equivalent:

(a) There exists a linear operator $T \in L(X, Y)$ such that the following applies:

$$
T_{1}(x) \leq T(x) \leq T_{2}(x), x \in X_{+}, T\left(x_{j}\right)=y_{j}, j \in J ;
$$

(b) For any finite subset $J_{0} \subset J$ and any $\left\{\lambda_{j}\right\}_{j \in J_{0}} \subset \mathbb{R}$, the following implication holds true:

$$
\left(\sum_{j \in J_{0}} \lambda_{j} x_{j}=\psi_{2}-\psi_{1}, \psi_{1}, \psi_{2} \in X_{+}\right) \Longrightarrow \sum_{j \in J_{0}} \lambda_{j} y_{j} \leq T_{2}\left(\psi_{2}\right)-T_{1}\left(\psi_{1}\right) ;
$$

If $X$ is a vector lattice, then assertions (a) and (b) are equivalent to (c), where the following applies:

(c) $\quad T_{1}(w) \leq T_{2}(w)$ for all $w \in X_{+}$and for any finite subset $J_{0} \subset J$ and $\forall\left\{\lambda_{j} ; j \in J_{0}\right\} \subset \mathbb{R}$, we have the following:

$$
\sum_{j \in J_{0}} \lambda_{j} y_{j} \leq T_{2}\left(\left(\sum_{j \in J_{0}} \lambda_{j} x_{j}\right)^{+}\right)-T_{1}\left(\left(\sum_{j \in J_{0}} \lambda_{j} x_{j}\right)^{-}\right) .
$$

For more general extension types and controlled regularity of linear operators see $[20,34]$ and the references given there. 


\subsection{Polynomial Approximation on Unbounded Subsets}

Our first polynomial approximation result on an unbounded interval was published in [33] Lemma 1.4. The motivation was solving Markov moment problems on $[0, \infty)$. We now complete the proof of this result presented as Lemma 1 below, since the original proof from [33] was incomplete. A second main polynomial approximation result is a very general one (see [36] Lemma 7 and [37] Lemma 3). Polynomial approximation and the expressions of nonnegative polynomials on unbounded intervals lead to characterization of the existence of the unique solutions for some multidimensional Markov moment problems in terms of quadratic forms with scalar or vector coefficients. The method works for Cartesian products of closed unbounded intervals, in particular for Markov moment problems on $\mathbb{R}^{n}, \mathbb{R}_{+}^{n}, n \in\{2,3, \ldots\}$.

\subsection{Elements of Self-Adjoint Operator Theory and Symmetric Matrices}

As it is well known, the real vector space $\mathcal{A}$ of all self-adjoint operators acting on an arbitrary complex or real Hilbert space $H$ is an ordered Banach space that is not a lattice. However, for any $A \in \mathcal{A}$, the subspace $Y(A)$ defined below by (3) is an order complete Banach lattice (and a commutative algebra) (see [4]). In particular, Theorem 3 and Corollary 1 hold $Y=Y(A)$ as a codomain space of the operator solution. On the other hand, symmetric matrices and some of their basic properties appear naturally in Theorem 5 of the present paper, where a polynomial solution for the truncated multidimensional moment problem is proposed. Evaluating the Euclidean norm of the vector formed by the coefficients of the solution is emphasized (without computing the involved its coefficients). Here, basic results on the spectrum of a positive definite symmetric matrix are applied.

\section{Results}

\subsection{Polynomial Approximation and Markov Moment Problem}

We start with polynomial approximation on $\mathbb{R}_{+}$, completing the proof of a result published in [33].

Lemma 1. Let $\psi: \mathbb{R}_{+}=[0, \infty) \rightarrow \mathbb{R}_{+}$be a continuous function, such that $\lim _{t \rightarrow \infty} \psi(t)$ exists in $\mathbb{R}_{+}$. Then there is a decreasing sequence $\left(h_{l}\right)_{l}$ in $\operatorname{Span}\left\{e_{k} ; k \in \mathbb{N}\right\}$, where the following applies:

$$
e_{k}(t)=\exp (-k t), k \in \mathbb{N}, t \in[0, \infty),
$$

such that $h_{l}(t) \geq \psi(t), t \geq 0, l \in \mathbb{N}=\{0,1,2, \ldots\}$, limh $_{l}=$ quniformly on $[0, \infty)$. There exists a sequence of polynomial functions $\left(\widetilde{p}_{l}\right)_{l \in \mathbb{N}}, \widetilde{p}_{l} \geq h_{l} \geq \psi$, lim $\widetilde{p}_{l}=\psi$, uniformly on compact subsets of $[0, \infty)$. In particular, such polynomial approximation holds for nonnegative continuous functions $\psi: \mathbb{R}_{+} \rightarrow \mathbb{R}_{+}$, having compact support.

Proof. The idea is to consider the sub-algebra $\hat{S}=\operatorname{Span}\left\{\hat{e}_{k} ; k \geq 0\right\}$ of $C([0, \infty])$, where $[0, \infty]$ is the Alexandroff extension of $[0, \infty)$, and $\hat{e}_{k}$ is the continuous extension of $e_{k}$ to $[0, \infty], \hat{e}_{k}(\infty)=0, k \geq 1, \hat{e}_{0}(\infty)=1$. This sub-algebra clearly separates the points of $[0, \infty]$ and contains the constant functions. According to Stone-Weierstrass theorem, $\hat{S}$ is dense in $C([0, \infty])$. It results that any continuous function $\psi: \mathbb{R}_{+} \rightarrow \mathbb{R}_{+}$, with the property that the limit $\lim _{t \rightarrow \infty} \psi(t)$ exists in $\mathbb{R}_{+}$, can be uniformly approximated on $\mathbb{R}_{+}$by elements from $\operatorname{Span}\left\{e_{k} ; k \geq 0\right\}$. As is well known, when the convergence is uniform, the approximating sequence $\left(h_{l}\right)_{l}$ for $\psi$ can be chosen such that $h_{l}(t) \geq \psi(t) \geq 0$ for all $t \in[0, \infty)$. Assume the following:

$$
h_{l}=\sum_{k=0}^{m_{l}} \alpha_{l, k} e_{k}, \alpha_{l, k} \in \mathbb{R}, l=0,1,2, \ldots
$$

If $\alpha_{l, k} \geq 0$, we obtain $\alpha_{l, k} e_{k} \leq \alpha_{l, k} p_{l, k}$, where $p_{l, k}$ is a majorizing partial sum of the power series of $e_{k}, e_{k}=\lim _{l \rightarrow \infty} p_{l, k}$, the convergence being uniform on any compact subset of $\mathbb{R}_{+}$. If $\alpha_{l, k}<0$, we deduce $\alpha_{l, k} e_{k}<\alpha_{l, k} q_{l, k}$, where $q_{l, k}$ is a minorizing partial sum of 
the power series of $e_{k}=\lim _{l \rightarrow \infty} p_{l, k}$, and the convergence is uniform on compact subsets of the nonnegative semi-axes. Summing as $k=0,1, \ldots, m_{l}$, one obtains a polynomial $\widetilde{p}_{l} \geq h_{l} \geq \psi \geq 0$ on $\mathbb{R}_{+}$. Since the sum defining $\widetilde{p}_{l}$ has a finite number of terms of such partial sums, we conclude $\widetilde{p}_{l} \rightarrow \psi$ uniformly on compact subsets of $\mathbb{R}_{+}$, as $l \rightarrow \infty$. This ends the proof.

In applications, the preceding lemma could be useful in order to prove a similar type result for continuous functions defined only on a compact subset $K \subset \mathbb{R}_{+}$, taking values in $\mathbb{R}_{+}$. For such a function $\varphi: K \rightarrow \mathbb{R}_{+}$, one denotes by $\varphi_{0}: \mathbb{R}_{+} \rightarrow \mathbb{R}_{+}$the extension of $\varphi$, which satisfies $\varphi_{0}(t)=0$ for all $t \in \mathbb{R}_{+} \backslash K$. With these notation, from Lemma 1 we infer the next result:

Lemma 2. Let $K \subset \mathbb{R}_{+}$be a compact subset and $\varphi: K \rightarrow \mathbb{R}_{+}$a continuous function. Then there exists a sequence $\left(\widetilde{p}_{l}\right)_{l \in \mathbb{N}}$ of polynomial functions, such that $\tilde{p}_{l} \geq \varphi_{0}$ on $\mathbb{R}_{+},\left.\widetilde{p}_{l}\right|_{K} \rightarrow \varphi, l \rightarrow \infty$, uniformly on $K$.

Proof. The idea is to reduce the proof to that of the preceding Lemma 1. Namely, we easily construct a continuous extension $\psi: \mathbb{R}_{+} \rightarrow \mathbb{R}_{+}$of $\varphi$, having compact support $\operatorname{supp}(\psi), \psi \geq \varphi_{0}$. Assuming this is done, if $\left(\widetilde{p}_{l}\right)_{l \in \mathbb{N}}$ are as in Lemma 1 , since $\widetilde{p}_{l} \rightarrow \psi$ uniformly on the compact $K$ and $\psi(t)=\varphi(t)$ for all $t$ in $K$, it results in the following:

$$
\sup _{t \in K}\left|\widetilde{p}_{l}(t)-\varphi(t)\right|=\sup _{t \in K}\left|\widetilde{p}_{l}(t)-\psi(t)\right| \rightarrow 0, l \rightarrow \infty .
$$

Moreover, according to Lemma 1 , we have $\widetilde{p}_{l} \geq \psi \geq \varphi_{0} \geq 0$ on $\mathbb{R}_{+}$. This will end the proof. To construct $\psi$, let $a=\operatorname{infK}, b=\operatorname{supK}, 0 \leq a \leq b<\infty$. It is clear that $\varphi_{0}$ might have discontinuities at the ends of the intervals representing connected components of $[0, \infty) \backslash K$. If $\varphi(b)=0$, then $\varphi_{0}$ is continuous at $b$ and on the entire interval $[b, \infty)$. If $\varphi(b)>0$, for an arbitrary $\varepsilon>0$, define $\psi$ on the interval $[b, b+\varepsilon]$ as the affine function whose graph is the line segment joining the points $(b, \varphi(b))$ and $(b+\varepsilon, 0), \psi(t)=0$ for all $t>b+\varepsilon, \psi(t)=\varphi(t)$ for all $t$ in $K$. It remains to define $\psi$ on each bounded connected component of $[0, \infty) \backslash K$. Let $\left(t_{1}, t_{2}\right)$ be such an interval, $t_{1}, t_{2} \in K, t_{1}<t_{2}$ and $0<\varepsilon<\left(t_{2}-t_{1}\right) / 2$. On the interval $\left[t_{2}-\varepsilon, t_{2}\right]$, we define $\psi$ as the affine function whose graph is the line segment of ends $\left(t_{2}-\varepsilon, 0\right),\left(t_{2}, \varphi\left(t_{2}\right)\right)$. Similarly, on the interval $\left[t_{1}, t_{1}+\varepsilon\right]$, we consider the line segment joining the points $\left(t_{1}, \varphi\left(t_{1}\right)\right),\left(t_{1}+\varepsilon, 0\right)$. The definition at points $t_{1}, t_{2}$ is in accordance with the previous condition and $\psi(t)=\varphi(t)$ for all $t \in K$. On the interval $\left(t_{1}+\varepsilon, t_{2}-\varepsilon\right), \psi \equiv 0$. Finally, if $a>0$ and $\varphi(a)>0$, taking $0<\varepsilon<a$, we define $\psi$ on the interval $[a-\varepsilon, a]$ as being the function whose graph is the line segment joining the points $(a-\varepsilon, 0),(a, \varphi(a)), \psi \equiv 0$ on $[0, a-\varepsilon]$. If $a=0$, we have $\psi(a)=\varphi(a)$, since $a \in K$ and the interval $(0, a)$ is empty. If $a>0$ and $\varphi(a)=0$, we define $\psi \equiv 0$ on $[0, a]$. Thus $\psi$ is defined, non-negative, continuous on $[0, \infty),\left.\psi\right|_{K} \equiv \varphi$, and $\operatorname{supp}(\psi)$ is compact, contained in $[0, b+\varepsilon]$. The proof is complete.

In the sequel, we prove our first new theorem, motivated by its corollary (see Corollary 1 below). Let $K \subset \mathbb{R}_{+}$be an arbitrary compact subset. We denote, by $X=C(K)$, the Banach lattice of all real-valued continuous functions on $K$, and let $Y$ be an arbitrary order complete Banach lattice. One denotes the following:

$$
\varphi_{j}(t)=t^{j}, t \in \mathbb{R}_{+}, j \in \mathbb{N} .
$$

Theorem 3. Let $T_{1}, T_{2}$ be two linear operators from $X$ to $Y$, such that $0 \leq T_{1} \leq T_{2}$ on the positive cone of $X$, and $\left(y_{n}\right)_{n \geq 0}$ a given sequence of elements in $Y$. The following statements are equivalent:

(a) There exists a unique (bounded) linear operator $T: X \rightarrow Y$, such that $T\left(\varphi_{j}\right)=y_{j}, j \in$ $\mathbb{N}, T_{1} \leq T \leq T_{2}$ on the positive cone of $X,\left\|T_{1}\right\| \leq\|T\| \leq\left\|T_{2}\right\| ;$ 
(b) For any polynomial $\sum_{j=0}^{m} \alpha_{j} \varphi_{j} \geq 0$ on $K$, we have $\sum_{j=0}^{m} \alpha_{j} T_{1}\left(\varphi_{j}\right) \leq \sum_{j=0}^{m} \alpha_{j} y_{j}$; if $J_{0} \subset \mathbb{N}$ is a finite subset and $\left\{\lambda_{j} ; j \in J_{0}\right\} \subset \mathbb{R}$, then the following applies:

$$
\sum_{i, j \in J_{0}} \lambda_{i} \lambda_{j} y_{i+j+l} \leq \sum_{i, j \in J_{0}} \lambda_{i} \lambda_{j} T_{2}\left(\varphi_{i+j+l}\right), l \in\{0,1\}
$$

(c) $T_{1} \leq T_{2}$ on $X_{+}$and for any polynomial $\sum_{j \in J_{0}} \lambda_{j} \varphi_{j}$, the following inequality holds:

$$
\sum_{j \in J_{0}} \lambda_{j} y_{j} \leq T_{2}\left(\left(\sum_{j \in J_{0}} \lambda_{j} \varphi_{j}\right)^{+}\right)-T_{1}\left(\left(\sum_{j \in J_{0}} \lambda_{j} \varphi_{j}\right)^{-}\right)
$$

Proof. According to the notations and assertions of (a), the implication of $(a) \Longrightarrow(b)$ is obvious. To prove the converse implication, we observe that first assertion of (b) says that defining the following:

$$
T_{0}\left(\sum_{j=0}^{m} \alpha_{j} \varphi_{j}\right)=\sum_{j=0}^{m} \alpha_{j} y_{j}, m \in \mathbb{N}, \alpha_{j} \in \mathbb{R},
$$

we obtain a linear operator defined on the subspace of polynomial functions, which verifies the moment conditions.

$$
T_{0}\left(\varphi_{j}\right)=y_{j}, j \in \mathbb{N},
$$

$T_{0} \geq T_{1}$ is on the convex cone $\mathcal{P}_{+}$of all polynomial functions, which are nonnegative on $K$. On the other hand, any element from $X=C(K)$ is dominated by a constant function, so that the subspace $\mathcal{P}$ of polynomial functions defined on $\mathbb{R}_{+}$verifies the hypothesis of Theorem 1, where $X_{1}$ stands for $X$, and $\mathcal{S}$ stands for $\mathcal{P}$. According to Theorem 1, the linear operator $T_{0}-T_{1}: \mathcal{P} \rightarrow Y$, which is positive on $\mathcal{P}_{+}=\mathcal{P} \cap X_{+}$, admits a positive linear extension $U: X \rightarrow Y$. We define $T=T_{1}+U \geq T_{1}$ on $X_{+}$. In addition $T \in L_{+}(X, Y)$ verifies the following:

$$
T\left(\varphi_{j}\right)=T_{1}\left(\varphi_{j}\right)+U\left(\varphi_{j}\right)=T_{1}\left(\varphi_{j}\right)+T_{0}\left(\varphi_{j}\right)-T_{1}\left(\varphi_{j}\right)=T_{0}\left(\varphi_{j}\right)=y_{j}, j \in \mathbb{N} .
$$

In other words, $T: X \rightarrow Y$ is a linear extension of $T_{0}: \mathcal{P} \rightarrow Y$, which dominates $T_{1}$ on $X_{+}$. Next, we prove that $T \leq T_{2}$ on $X_{+}$. To this end, observe that according to the second assertion of (b), we already know that $T \leq T_{2}$ on special polynomial functions, which are nonnegative on the entire semi axes $\mathbb{R}_{+}$. Indeed, any nonnegative polynomial $p=p(t)$ on $\mathbb{R}_{+}$has the explicit form $p(t)=q^{2}(t)+t r^{2}(t)$ for some On the other hand, since

$$
T \geq T_{1} \geq 0,
$$

the linear operator $T$ is positive and hence is also continuous; $T_{2}$ is continuous as well, thanks to its positivity. We now apply Lemma 2 for an arbitrary $\varphi \in X_{+}$. Using the notations of Lemma 2 and the above discussed assertions we infer the following:

$$
0 \leq T_{1}(\varphi) \leq T(\varphi)=\lim _{l \rightarrow \infty} T\left(\widetilde{p}_{l}\right) \leq \lim _{l \rightarrow \infty} T_{2}\left(\widetilde{p}_{l}\right)=T_{2}(\varphi), \varphi \in X_{+} .
$$

It remains to prove the last relation of (a). If $\psi$ is an arbitrary function in $X$, then (2) leads to the following:

$$
T(\psi) \leq T(|\psi|) \leq T_{2}(|\psi|)
$$

and similarly $-T(\psi)=T(-\psi) \leq T_{2}(|\psi|)$. These inequalities yield $|T(\psi)| \leq T_{2}(|\psi|)$ and, since $Y$ is a Banach lattice, the conclusion is $\|T(\psi)\| \leq\left\|T_{2}(|\psi|)\right\| \leq\left\|T_{2}\right\|\||\psi|\|=\left\|T_{2}\right\| \|$ $\psi \|, \psi$ in $X$. Thus, $\|T\| \leq\left\|T_{2}\right\|$. Similarly, $\left\|T_{1}\right\| \leq\|T\|$. The equivalence $(a) \Leftrightarrow(c)$ follows directly from Theorem 2 . This completes the proof. 
Next, we recall a well-known important example of an order complete Banach lattice $Y$ of self-adjoint operators acting on a complex or real Hilbert space $H$. Let $\mathcal{A}=\mathcal{A}(H)$ be the ordered vector space of all of the self-adjoint operators acting on $H$, and let $A \in \mathcal{A}$. The natural order relation on $\mathcal{A}$ is $A \leq B$ if and only if $\langle A h, h\rangle \leq\langle B h, h\rangle$ for all $h$ in $H$.

One can prove that $\mathcal{A}$ with this ordering is not a lattice. Therefore, it is interesting to fix $A \in \mathcal{A}$ and define the following:

$$
Y_{1}(A)=\{V \in \mathcal{A} ; A V=V A\}, Y(A)=\left\{V \in Y_{1}(A) ; W V=V W, \forall W \in Y_{1}(A)\right\} .
$$

Then, $Y(A)$ is an order complete Banach lattice (and a commutative real algebra), as discussed in [4]. If $V \in \mathcal{A}$, we denote by $\sigma(V)$ the spectrum of $V$ and by $d E_{V}$ the spectral measure attached to $V$.

Corollary 1. With the above notations, assume that $A$ is a positive self-adjoint operator acting on $H, Y(A)$ is the space defined by (3), and $\left(B_{j}\right)_{j \in \mathbb{N}}$ is a sequence of operators in $Y(A)$. The following statements are equivalent:

(a) There exists a unique positive linear operator $T: C(\sigma(A)) \rightarrow Y$, such that

$$
T\left(\varphi_{j}\right)=B_{j}, j \in \mathbb{N}, T(\varphi) \leq \int_{\sigma(A)} \varphi(t) d E_{A}, \varphi \in(C(\sigma(A)))_{+},\|T\| \leq 1
$$

(b) For any polynomial $\sum_{j=0}^{m} \alpha_{j} \varphi_{j} \geq 0$ on $\sigma(A)$, it results $\sum_{j=0}^{m} \alpha_{j} B_{j} \geq 0$; if $J_{0} \subset \mathbb{N}$ is a finite subset and $\left\{\lambda_{j} ; j \in J_{0}\right\} \subset \mathbb{R}$, then the following applies:

$$
\sum_{i, j \in J_{0}} \lambda_{i} \lambda_{j} B_{i+j+l} \leq \sum_{i, j \in J_{0}} \lambda_{i} \lambda_{j} A^{i+j+l}, l \in\{0,1\} .
$$

Proof. Since $A$ is self-adjoint and positive, its spectrum $\sigma(A)$ is a compact contained in $[0, \infty)$. One applies Theorem 3 for $K=\sigma(A), T_{2}: C(\sigma(A)) \rightarrow Y, T_{2}(\varphi)=\int_{\sigma(A)} \varphi(t) d E_{A}=$ $\varphi(A), T_{1}=0$.

Remark 1. It would be useful to know whether a similar result to that of Lemma 1 holds when we replace $\mathbb{R}_{+}$with $\mathbb{R}$. In this case, the dominating polynomials $\widetilde{p}_{l}$ should be nonnegative on the entire real axes, and hence would be the sums of squares. If $\psi: \mathbb{R} \rightarrow \mathbb{R}_{+}$would be continuous, compactly supported and even function, then the problem is reduced to that solved by Lemma 1; $\psi$ can be approximated by dominating even polynomials, the convergence holding uniformly on compact subsets of $\mathbb{R}$. If $\psi$ is not even, while the other assumptions on it are maintained, the polynomial approximation is not obvious.

Remark 2. In Theorem 3, the main implication is $(b) \Longrightarrow(a)$, since the conditions of $(b)$ are checkable in terms of the moments $y_{j}$ and the given operators $T_{1}, T_{2}$.

Remark 3. It would be interesting to prove results such as Lemmas 1 and 2 in several variables. Namely, being given a nonnegative continuous compactly supported real function $f$ defined on $\mathbb{R}_{+}^{n}, n \geq 2$, and denoting by $K$ its support, we could approximate $f$ on $K \subseteq K_{1} \times \cdots \times K_{n}$, where $K_{j}=\operatorname{pr}_{j}(K), j=1, \ldots, n$, by the sums of products $f_{1} \otimes \cdots \otimes f_{n}, f_{j}: K_{j} \rightarrow \mathbb{R}_{+}$is continuous for all $j=1, \ldots, n$, via the Stone-Weierstrass theorem or Bernstein polynomials of $n$ variables. Then, we could apply to each $f_{j}$ Lemma 2 , and finally obtain the approximation of $f$ by finite sums of products of polynomials $p_{1} \otimes \cdots \otimes p_{n}$, where $p_{j}: \mathbb{R}_{+} \rightarrow \mathbb{R}_{+}, p_{j} \geq f_{j}$ on $K_{j}, j=1, \ldots, n$, and the approximation holds uniformly on compact subsets of $\mathbb{R}_{+}^{n}$. The motivation for such consideration is that being given a system of commuting positive self-adjoint operators $A_{1}, \ldots A_{n}$ acting on a Hilbert space, we could try to prove a result similar to Corollary 1, by means of considering polynomial uniform approximation on $K=\sigma\left(A_{1}\right) \times \cdots \times \sigma\left(A_{1}\right)$, where $\sigma\left(A_{j}\right)$ is the spectrum of $A_{j}, j=1, \ldots n$. 
If $S \subseteq \mathbb{R}^{n}$ is an arbitrary closed unbounded subset, then we denote by $\mathcal{P}_{+}$the convex cone of all polynomial functions (with real coefficients), taking nonnegative values at any point of $S$. Also, $\mathcal{P}_{++}$will be a sub-cone of $\mathcal{P}_{+}$generated by special nonnegative polynomials expressible in terms of the sums of squares. One denotes by $C_{0}(S)$ the vector space of real-valued continuous and compactly supported functions defined on $S$.

Theorem 4. Let $S \subseteq \mathbb{R}^{n}$ be a closed unbounded subset, $v$ a positive Borel moment determinate measure on $S$, having finite moments of all orders, $X=L_{v}^{1}(S), \varphi_{j}(t)=t^{j}, t \in S, j \in \mathbb{N}^{n}$. Let $Y$ be an order complete Banach lattice, $\left(y_{j}\right)_{j \in \mathbb{N}^{n}}$ a given sequence of elements in $Y, T_{1}$, and $T_{2}$ two bounded linear operators from $X$ to $Y$. Assume that there exists a sub-cone $\mathcal{P}_{++} \subseteq \mathcal{P}_{+}$, such that each $f \in\left(C_{0}(S)\right)_{+}$can be approximated in $X$ by a sequence $\left(p_{l}\right)_{l}, p_{l} \in \mathcal{P}_{++}, p_{l} \geq f$ for all $l$. The following statements are equivalent:

(a) There exists a unique (bounded) linear operator $T: X \rightarrow Y, T\left(\varphi_{j}\right)=y_{j}, j \in \mathbb{N}^{n}, 0 \leq T_{1} \leq$ $T \leq T_{2}$ on $X_{+}$

$$
\left\|T_{1}\right\| \leq\|T\| \leq\left\|T_{2}\right\|
$$

(b) For any finite subset $J_{0} \subset \mathbb{N}^{n}$ and any $\left\{\lambda_{j} ; j \in J_{0}\right\} \subset \mathbb{R}$, the following implications hold true:

$$
\begin{gathered}
\sum_{j \in J_{0}} \lambda_{j} \varphi_{j} \in \mathcal{P}_{+} \Longrightarrow \sum_{j \in J_{0}} \lambda_{j} T_{1}\left(\varphi_{j}\right) \leq \sum_{j \in J_{0}} \lambda_{j} y_{j} \\
\sum_{j \in J_{0}} \lambda_{j} \varphi_{j} \in \mathcal{P}_{++} \Longrightarrow \sum_{j \in J_{0}} \lambda_{j} T_{1}\left(\varphi_{j}\right) \geq 0, \sum_{j \in J_{0}} \lambda_{j} y_{j} \leq \sum_{j \in J_{0}} \lambda_{j} T_{2}\left(\varphi_{j}\right) .
\end{gathered}
$$

Proof. We start by observing that the first condition (5) implies the positivity of the bounded linear operator $T_{1}$ via its continuity. Indeed, if $f \in\left(C_{0}(S)\right)_{+}, p_{l} \in \mathcal{P}_{++}, p_{l} \geq f$ for all $l, p_{l} \rightarrow f$ in $L_{v}^{1}(S)$, then, according to the first condition (5), $T_{1}\left(p_{l}\right) \geq 0$ for all $l \in \mathbb{N}$ and the continuity of $T_{1}$ yields the following:

$$
T_{1}(f)=\lim _{l} T_{1}\left(p_{l}\right) \geq 0
$$

Since $\left(C_{0}(S)\right)_{+}$is dense in $X_{+}$via measure theory reasons, the continuity of $T_{1}$ implies $T_{1} \geq 0$ on $X_{+}$. Thus, $T_{1}$ is a positive linear operator. Next, we define $T_{0}: \mathcal{P} \rightarrow Y$, $T_{0}\left(\sum_{j \in J_{0}} \lambda_{j} \varphi_{j}\right)=\sum_{j \in J_{0}} \lambda_{j} y_{j}$, where the sums are finite and the coefficients $\lambda_{j}$ are arbitrary real numbers. Condition (4) says that $T_{0}-T_{1} \geq 0$ on $\mathcal{P}_{+}$. If we consider the vector subspace $X_{1}$ of $X$ formed by all functions $\psi \in X$ having the modulus $|\psi|$ dominated by a polynomial $p \in \mathcal{P}_{+}$on the entire set $S$, then $\mathcal{P}$ is a majorizing subspace of $X_{1}$ and $T_{0}-T_{1}$ is a positive linear operator on $\mathcal{P}$. The application of Theorem 1 leads to the existence of a positive linear extension $U: X_{1} \rightarrow Y$, of $T_{0}-T_{1}$. Obviously, $X_{1}$ contains $C_{0}(S)+\mathcal{P}:$ $\left(p \in \mathcal{P} \Longrightarrow|p|=\sqrt{1 \cdot p^{2}} \leq\left(1+p^{2}\right) / 20\right.$ the $f$ as the preceding one $\left.\in \mathcal{P}\right)$. Indeed, since $\varphi \in C_{0}(S) \Longrightarrow|\varphi| \in\left(C_{0}(S)\right)_{+} \Longrightarrow|\varphi| \leq b 1 \in \mathcal{P}$ (according to Weierstrass' Theorem), we infer that $\varphi \in X_{1}$; here, $b<\infty$ is a real number. Hence, $C_{0}(S) \subset X_{1}$. Now let $p \in \mathcal{P}$, we observe the following:

$$
1+p^{2}-2|p|=(1-|p|)^{2} \geq 0
$$

which can be written as follows:

$$
|p| \leq \frac{1+p^{2}}{2} \in \mathcal{P}
$$

According to the definition of $X_{1}$, it results in $\mathcal{P} \subset X_{1}$. Consequently, $C_{0}(S)+\mathcal{P} \subset X_{1}$. Going back to the positive linear extension $U: X_{1} \rightarrow Y$, of $T_{0}-T_{1}$, we conclude that 
$\hat{T}_{0}=U+T_{1}: X_{1} \rightarrow Y$ is an extension of $T_{0}, \hat{T}_{0} \geq T_{1}$ on $\left(X_{1}\right)_{+}$, and $\hat{T}_{0}(p)=T_{0}(p) \leq T_{2}(p)$ for all $p \in \mathcal{P}_{++}$, according to the last requirement (5). A first conclusion is as follows:

$$
T_{1}(p) \leq \hat{T}_{0}(p) \leq T_{2}(p) \text { for all } p \in \mathcal{P}_{++}, \hat{T}_{0}(\psi) \geq T_{1}(\psi) \geq 0, \psi \in\left(X_{1}\right)_{+} .
$$

Our next goal is to prove the continuity of $\hat{T}_{0}$ on $C_{0}(S)$. Let $\left(f_{l}\right)_{l>0}$ be a sequence of nonnegative continuous compactly supported functions, such that $\bar{f}_{l} \rightarrow 0$ in $X_{1}$, and take a sequence of polynomials $p_{l} \geq f_{l} \geq 0, p_{l} \in \mathcal{P}_{++}$for all $l$, such that the following convergence result holds: $\left\|p_{l}-f_{l}\right\|_{1} \rightarrow 0, l \rightarrow \infty$. Then apply the following:

$$
\left\|p_{l}\right\|_{1} \leq\left\|p_{l}-f_{l}\right\|_{1}+\left\|f_{l}\right\|_{1} \rightarrow 0, l \rightarrow \infty .
$$

Now (6) and the continuity of $T_{1}, T_{2}$, yield the following:

$$
0 \leftarrow T_{1}\left(p_{l}\right) \leq \hat{T}_{0}\left(p_{l}\right) \leq T_{2}\left(p_{l}\right) \rightarrow 0,
$$

hence $\hat{T}_{0}\left(p_{l}\right) \rightarrow 0$. It results in the following:

$$
0 \leq T_{1}\left(f_{l}\right) \leq \hat{T}_{0}\left(f_{l}\right) \leq \hat{T}_{0}\left(p_{l}\right) \rightarrow 0 .
$$

Hence, $\hat{T}_{0}\left(f_{l}\right) \rightarrow 0$. If $\left(g_{n}\right)_{n \geq 0}$ is an arbitrary sequence of compactly supported and continuous functions, such as that of $g_{n} \rightarrow 0$ in $X_{1}$, then $g_{n}^{+} \rightarrow 0, g_{n}^{-} \rightarrow 0$. According to what we already have proved, we can write $\hat{T}_{0}\left(g_{n}^{+}\right) \rightarrow 0$ and $\hat{T}_{0}\left(g_{n}^{-}\right) \rightarrow 0$, which further yield $\hat{T}_{0}\left(g_{n}\right) \rightarrow 0$. This proves the continuity of $\hat{T}_{0}$ on $C_{0}(S)$, and the subspace $C_{0}(S)$ is dense in $X$. Hence, there exists a unique continuous linear extension $T \in B(X, Y)$ of $\hat{T}_{0}$. It results in $0 \leq T_{1} \leq T \leq T_{2}$ on $X_{+},\left\|T_{1}\right\| \leq\|T\| \leq\left\|T_{2}\right\|, T\left(\varphi_{j}\right)=T_{0}\left(\varphi_{j}\right)=y_{j}, j \in \mathbb{N}^{n}$. Indeed, $T_{1}, T, T_{2}$ are linear and continuous, and $\mathcal{P}_{++}$is dense in $\left(C_{0}(S)\right)_{+}$, hence it is dense in $X_{+}$as well. For an arbitrary $\varphi \in X$, the following inequalities hold true, via the preceding remarks:

$$
\begin{gathered}
\pm T(\varphi)=T( \pm \varphi) \leq T(|\varphi|) \leq T_{2}(|\varphi|) \Longrightarrow \\
|T(\varphi)| \leq T_{2}(|\varphi|) \Longrightarrow\|T(\varphi)\| \leq\left\|T_{2}(|\varphi|)\right\| \leq\left\|T_{2}\right\|\|\varphi\| .
\end{gathered}
$$

It follows that $\|T\| \leq\left\|T_{2}\right\|$, and similarly, $\left\|T_{1}\right\| \leq\|T\|$. The uniqueness of the solution $T$ follows from the density of the polynomials in $X$, via the continuity of the linear operator $T$. This ends the proof.

Our next goal is to give a result for the Markov moment problem in the space $L_{v}^{1}(\mathbb{R})$, where $v$ is a moment determinate measure on $\mathbb{R}$, with finite moments $\int_{\mathbb{R}} t^{k} d v \in \mathbb{R}$ of all orders $k \in \mathbb{N}$.

Corollary 2. Let $X=L_{v}^{1}(\mathbb{R})$, wherev is a moment determinate positive Borel measure on $\mathbb{R}$, with finite moments of all orders. Assume that $Y$ is an arbitrary order complete Banach lattice, and $\left(y_{n}\right)_{n \geq 0}$ is a given sequence having its terms in $Y$. Let $T_{1}, T_{2}$ be two linear operators from $X$ to $Y$, such that $0 \leq T_{1} \leq T_{2}$ on $X_{+}$. The following statements are equivalent:

(a) There exists a unique bounded linear operator $T$ from $X$ to $Y, T_{1} \leq T \leq T_{2}$ on $X_{+}$, $\left\|T_{1}\right\| \leq\|T\| \leq\left\|T_{2}\right\|$, such that $T\left(\varphi_{n}\right)=y_{n}$ for all $n \in \mathbb{N}$;

(b) If $J_{0} \subset \mathbb{N}$ is a finite subset and $\left\{\lambda_{j} ; j \in J_{0}\right\} \subset \mathbb{R}$, then the following applies:

$$
\sum_{i, j \in J_{0}} \lambda_{i} \lambda_{j} T_{1}\left(\varphi_{i+j}\right) \leq \sum_{i, j \in J_{0}} \lambda_{i} \lambda_{j} y_{i+j} \leq \sum_{i, j \in J_{0}} \lambda_{i} \lambda_{j} T_{2}\left(\varphi_{i+j}\right) .
$$

In the case of Corollary 2, we have $\mathcal{P}_{++}=\mathcal{P}_{+}$. Going further to the multidimensional case, for examples of such sub-cones $\mathcal{P}_{++}$of $\mathcal{P}_{+}$and their applications on the Markov moment problem, see [37] Theorems 5 and 6. Namely, these theorems emphasize the importance of using quadratic forms in the multidimensional case, when nonnegative 
polynomials are not usually expressible as sums of squares. In both of these examples, the inclusion $\mathcal{P}_{++} \subset \mathcal{P}_{+}$is strict. In the case of $S=\mathbb{R}_{+}^{n}$ (respectively, $S=\mathbb{R}^{n}$ ), $n \geq 2$, the cone $\mathcal{P}_{++}$consists in all polynomials that are sums of products of the form.

$$
p_{1} \otimes \cdots \otimes p_{n}
$$

where each $p_{j}, j=1, \ldots, n$, is a nonnegative polynomial on $\mathbb{R}_{+}$(respectively, on $\mathbb{R}$ ), hence is expressible by means of sums of squares of polynomials of one variable. Proceeding this way, the conditions of (5) can be written in terms of quadratic forms (see Corollary 3 stated below).

Corollary 3. Let $v=v_{1} \times \cdots \times v_{n}, n \geq 2, v_{j}$ be an $M$-determinate (moment determinate) positive regular Borel measure on $\mathbb{R}, j=1, \ldots, n, X=L_{v}^{1}\left(\mathbb{R}^{n}\right), \varphi_{j}(t)=t^{j}, t \in \mathbb{R}^{n}, j \in \mathbb{N}^{n}$. Additionally, assume that $v_{j}$ has finite moments of all orders, $j=1, \ldots, n$. Let $Y$ be an order complete Banach lattice, $\left(y_{j}\right)_{j \in \mathbb{N}^{n}}$ a given sequence of elements in $Y, T_{1}$, and $T_{2}$ two bounded linear operators from $X$ to $Y$. The following statements are equivalent:

(a) There exists a unique (bounded) linear operator $T: X \rightarrow Y, T\left(\varphi_{j}\right)=y_{j}, j \in \mathbb{N}^{n}, 0 \leq T_{1} \leq$ $T \leq T_{2}$ on $X_{+}$,

$$
\left\|T_{1}\right\| \leq\|T\| \leq\left\|T_{2}\right\| ;
$$

(b) For any finite subset $J_{0} \subset \mathbb{N}^{n}$ and any $\left\{\lambda_{j} ; j \in J_{0}\right\} \subset \mathbb{R}$, the following implication holds true:

$$
\sum_{j \in J_{0}} \lambda_{j} \varphi_{j} \in \mathcal{P}_{+} \Longrightarrow \sum_{j \in J_{0}} \lambda_{j} T_{1}\left(\varphi_{j}\right) \leq \sum_{j \in J_{0}} \lambda_{j} y_{j} ;
$$

for any finite subsets $J_{k} \subset \mathbb{N}, k=1, \ldots, n$, and any $\left\{\lambda_{j_{k}}\right\}_{j_{k} \in J_{k}} \subset \mathbb{R}$, the following inequalities hold:

$$
\begin{aligned}
0 \leq & \sum_{i_{1}, j_{1} \in J_{1}}\left(\cdots\left(\sum_{i_{n} . j_{n} \in J_{n}} \lambda_{i_{1}} \lambda_{j_{1}} \cdots \lambda_{i_{n}} \lambda_{j_{n}} T_{1}\left(\varphi_{i_{1}+j_{1}, \ldots, i_{n}+j_{n}}\right)\right) \cdots\right) ; \\
& \sum_{i_{1}, j_{1} \in J_{1}}\left(\cdots\left(\sum_{i_{n} \cdot j_{n} \in J_{n}} \lambda_{i_{1}} \lambda_{j_{1}} \cdots \lambda_{i_{n}} \lambda_{j_{n}} y_{i_{1}+j_{1} \ldots, i_{n}+j_{n}}\right) \cdots\right) \\
\leq & \sum_{i_{1}, j_{1} \in J_{1}}\left(\cdots\left(\sum_{i_{n} \cdot j_{n} \in J_{n}} \lambda_{i_{1}} \lambda_{j_{1}} \cdots \lambda_{i_{n}} \lambda_{j_{n}} T_{2}\left(\varphi_{i_{1}+j_{1}, \ldots, i_{n}+j_{n}}\right)\right) \cdots\right) .
\end{aligned}
$$

\subsection{On a Polynomial Solution for Truncated Multidimensional Moment Problem}

In the end of this subsection, we propose a polynomial solution for the truncated multidimensional scalar-valued moment problem, completing a result of [38]. The related evaluation for the norm of the vector formed with the coefficients of the polynomial solution is outlined. The general idea is to replace the space $\mathcal{P}$ of all of the polynomial functions on a closed subset $S \subseteq \mathbb{R}^{n}, n \in\{2,3, \ldots\}$ having nonempty interior, with the subspace generated by the following monomials:

$$
\varphi_{j}(t)=t^{j}=t_{1}^{j_{1}} \cdots t_{n}^{j_{n}}, t=\left(t_{1}, \ldots, t_{n}\right) \in S, j=\left(j_{1}, \ldots, j_{n}\right), j_{k} \in\{0,1, \ldots, d\},
$$

where $k=1, \ldots, n$, where $d \geq 1$ is a fixed integer. Let $w$ be a continuous positive real valued function on $S$, such that all the absolute moments,

$$
\int_{S}|t|^{j} w(t) d t, j=\left(j_{1}, \ldots, j_{k}, \ldots, j_{n}\right) \in \mathbb{N}^{n}, 0 \leq j_{k} \leq d, k=1, \ldots, n,
$$


are finite (here $|t|^{j}=\left|t_{1}\right|^{j_{1}} \cdots\left|t_{n}\right|^{j_{n}}, d t=d t_{1} \cdots d t_{n}$ ). Being given a finite set of numbers $\left\{y_{j} ; j \in \mathbb{N}^{n}, j_{k} \leq d, k=1, \ldots, n\right\}$, we are looking for a solution $h$ of the moment interpolation problem, as follows:

$$
\int_{S} t^{j} h(t) w(t) d t=y_{j}, 0 \leq j_{k} \leq d, k=1, \ldots, n .
$$

The simplest function $h$ satisfying equalities (7) is the following polynomial:

$$
h(t)=\sum_{\substack{0 \leq l_{k} \leq d, k=1, \ldots, n}} \lambda_{l} t^{l}, t \in S,
$$

where $l=\left(l_{1}, \ldots, l_{k}, \ldots, l_{n}\right) \in \mathbb{N}^{n}$. The number of terms of the sum in (8) is at most $N=(d+1)^{n}$, and $N$ is the dimension of the subspace of polynomials involved in this problem. We have to determine the unknown coefficients $\lambda_{l}, 0 \leq l_{k} \leq d, k=1, \ldots, n$, such that $h$ be a solution of (7). The corresponding result gives the explicit form of the solution and related evaluations of its norm. These inequalities do not involve computing the inverse of the matrix $A$ (see the proof of the next theorem). Theorem 5 is formulated as follows:

Theorem 5. The vector $\lambda=\left(\lambda_{j}\right)_{\substack{0 \leq j_{k} \leq d, 1 \leq k \leq n}}$ of the unknowns $\lambda_{l}, 0 \leq l_{k} \leq d, k=1, \ldots, n$ is defined by (7) and (8) and is given by (11), where the matrix $A$ is defined by (10). This matrix is positive definite and evaluations (12) and (13) hold.

Proof. Inserting $h(t)$, defined by (8) in (7), it is easy to see that the necessary and sufficient conditions required on $\lambda_{l}, l_{k} \leq d, k=1, \ldots, n$ are as follows:

$$
\sum_{\substack{0 \leq l_{k} \leq d, k=1, \ldots, n}} \lambda_{l} \int_{S} t^{j+l} w(t) d t=y_{j}, j_{k} \leq d, k=1, \ldots, n .
$$

This is a linear system with the unknowns $\lambda_{l}, 0 \leq l_{k} \leq d, k=1, \ldots, n, l=\left(l_{1}, \ldots, l_{n}\right)$, which consisted of equations with $N$ unknowns.

$$
N=(d+1)^{n}
$$

The matrix of the system is the $N \times N$ symmetric matrix.

$$
A=\left(a_{j, l}\right)_{0 \leq j_{k}, l_{k} \leq d^{\prime}}, a_{j, l}=\int_{S} t^{j+l} w(t) d t, 0 \leq j_{k} \leq d, 0 \leq l_{k} \leq d, k=1, \ldots, n .
$$

The main property of matrix $A$ is that it is positive definite. Indeed, according to (10), the following relations hold true:

$$
\sum_{\substack{0 \leq j_{k} \leq d, 0 \leq l_{k} \leq d}} a_{j, l} \lambda_{j} \lambda_{l}=\int_{S}\left(\sum_{0 \leq j_{k} \leq d} \lambda_{j} t^{j}\right)^{2} w(t) d t>0
$$

for all not null vectors $\lambda=\left(\lambda_{j}\right)_{\substack{0 \leq j_{k} \leq d, 1 \leq k \leq n}}$. As a consequence, all eigenvalues of matrix $A$ are positive. In particular, 0 is not in the spectrum of $A$, so that this matrix is invertible and its inverse is also positive definite. Since (9) can be written as follows:

$$
A \lambda^{t}=y^{t},
$$


we infer the following unique solution:

$$
\lambda^{t}=A^{-1} \boldsymbol{y}^{t}
$$

Hence, the problem is reduced to the computation of $A^{-1}$. A related evaluation might be useful. For example, if we denote by $\Omega_{A}$ the greatest eigenvalue and by $\omega_{A}$ the smallest eigenvalue of $A$, then the following applies:

$$
\|\lambda\|_{2} \leq\left\|A^{-1}\right\|\|\boldsymbol{y}\|_{2}=\left(1 / \omega_{A}\right)\|\boldsymbol{y}\|_{2},
$$

where \|\|$_{2}$ is the Euclidean norm on $\mathbb{R}^{N}$. Similarly, from the following inequality:

$$
A^{-1} \succcurlyeq\left(1 / \Omega_{A}\right) I,
$$

also using (11) and the Cauchy-Schwarz inequality, the following evaluations hold:

$$
\|\lambda\|_{2}\|y\|_{2} \geq\langle\lambda, y\rangle=\left\langle A^{-1} \boldsymbol{y}, \boldsymbol{y}\right\rangle \geq\left(1 / \Omega_{A}\right)\|\boldsymbol{y}\|_{2}^{2} .
$$

It results in the following:

$$
\|\lambda\|_{2} \geq\left(1 / \Omega_{A}\right)\|\boldsymbol{y}\|_{2} .
$$

The proof is complete.

\section{Discussion}

The first part of Section 3 is completely devoted to characterizing the existence and uniqueness of the solution for a class of the Markov moment problem, also controlling the norm of the solution. The necessary and sufficient conditions are written in terms of the moment sequences. The domain is an $L_{v}^{1}(S)$ space, where $S$ is a closed subset of $\mathbb{R}^{n}, n \geq 1$, and $v$ is a moment determinate positive regular Borel measure on $S$, with finite absolute moments of all order. For such measures, the nonnegative polynomials are dense in $\left(L_{v}^{1}(S)\right)_{+}$. The case when the domain is $C(K)$, the Banach lattice of all real-valued continuous functions defined on the compact subset $K \subset \mathbb{R}_{+}$, is also under attention. Here, the novelty is the approximation of the nonnegative elements of $C(K)$, by dominating polynomials that are nonnegative on the entire semi axes $\mathbb{R}_{+}$. Therefore, the second condition of (b), Theorem 3, can be formulated in terms of quadratic forms. The motivations for considering such problems are mentioned. Namely, $K$ could be the spectrum of a positive self-adjoint operator (see Corollary 1). Besides a well-known old result on the extension of linear positive operators preserving positivity (Theorem 1), polynomial approximation results on unbounded subsets are also used. These methods allow controlling not only the positivity (which implies its continuity), but also the norm of the linear solution $T$. This is the reason for considering the codomain $Y$ as an order complete Banach lattice, not only an order complete Banach space. One solves, partially, the difficulty arising from the fact that on $\mathbb{R}^{n}, n \geq 2$ there exists nonnegative polynomials that are not sums of squares. Theorem 5 proposes a polynomial solution for the truncated moment problem. The norm of the vector of the coefficients of this solution is evaluated without computing effectively the coefficients. This method could work for the full moment problem, where a real analytic solution should replace the polynomial solution of the truncated problem. This can be a direction for future work.

\section{Conclusions}

In Section 3, it seems that the main results are as follows: Lemma 1, Theorem 3, the discussion outlined in Remark 3, Theorem 4, Corollaries 2, 3, and Theorem 5 . All of these results (except Theorem 5) are based on main non-trivial lemmas and theorems on polynomial approximation, recently reviewed in [37]. With respect to the paper [37], the new element of the present work is the condition $T \geq T_{1}$ on the positive cone of the domain 
space, where $T_{1}$ is not necessarily the null operator. On the other hand, Theorem 4 (whose proof is not trivial) works for any sub-cone $\mathcal{P}_{++}$, which verifies (5). Finally, Theorem 5 brings new elements, compared with first formulation of this idea sketched in [38].

Funding: This research received no external funding.

Institutional Review Board Statement: Not applicable.

Informed Consent Statement: Not applicable.

Data Availability Statement: Not applicable.

Conflicts of Interest: The author declares no conflict of interest.

\section{References}

1. Akhiezer, N.I. The Classical Moment Problem and Some Related Questions in Analysis; Oliver and Boyd: Edinburgh-London, UK, 1965.

2. Krein, M.G.; Nudelman, A.A. Markov Moment Problem and Extremal Problems; American Mathematical Society: Providence, RI, USA, 1977.

3. Schmüdgen, K. The Moment Problem. In Graduate Texts in Mathematics; Springer: Berlin/Heidelberg, Germany; New York, NY, USA, 2017. [CrossRef]

4. Cristescu, R. Ordered Vector Spaces and Linear Operators; Academiei, Bucharest, and Abacus Press: Tunbridge Wells, Kent, UK, 1976.

5. Niculescu, C.; Popa, N. Elements of Theory of Banach Spaces; Academiei: Bucharest, Romania, 1981. (In Romanian)

6. Choudary, A.D.R.; Niculescu, C.P. Real Analysis on Intervals; Springer: Berlin/Heidelberg, Germany; New York, NY, USA; Dordrecht, The Netherlands; London, UK; New Delhi, India, 2014. [CrossRef]

7. Niculescu, C.P.; Persson, L.-E. Convex Functions and Their Applications, A Contemporary Approach, 2nd ed.; (CMS Books in Mathematics); Springer: New York, NY, USA, 2018; Volume 23.

8. Boboc, N.; Bucur, G. Convex Cones of Continuous Functions on Compact Spaces; Academiei: Bucharest, Romania, 1976. (In Romanian)

9. Rudin, W. Real and Complex Analysis, 3rd ed.; McGraw-Hill Book Company: New York, NY, USA, 1987.

10. Berg, C.; Christensen, J.P.R.; Jensen, C.U. A remark on the multidimensional moment problem. Math. Ann. 1979, 243, 163-169. [CrossRef]

11. Berg, C.; Durán, A.J. The fixed point for a transformation of Hausdorff moment sequences and iteration of a rational function. Math. Scand. 2008, 103, 11-39. [CrossRef]

12. Berg, C.; Beygmohammadi, M. On a fixed point in the metric spece of normalized Hausdorff moment sequences. Rend. Circ. Mat. Palermo Ser. II 2010, 82 (Suppl. 82), 251-257.

13. Cassier, G. Problèmes des moments sur un compact de $\mathbb{R}^{n}$ et décomposition des polynmes à plusieurs variables (Moment problems on a compact subset of $\mathbb{R}^{n}$ and decomposition of polynomials of several variables). J. Funct. Anal. 1984, 58, 254-266. [CrossRef]

14. Choquet, G. Le problème des moments (The moment problem). In Séminaire d'Initiation à l'Analise; Institut H. Poincaré: Paris, France, 1962.

15. Fuglede, B. The multidimensional moment problem. Expo. Math. 1983, 1, 47-65.

16. Haviland, E.K. On the momentum problem for distributions in more than one dimension. Am. J. Math. 1936, 58, 164-168. [CrossRef]

17. Marshall, M. Polynomials non-negative on a strip. Proc. Am. Math. Soc. 2010, 138, 1559-1567. [CrossRef]

18. Inverardi, P.L.N.; Tagliani, A. Stieltjies and Hamburger reduced moment problem when MaxEnt solution does not exist. Mathematics 2021, 9, 309. [CrossRef]

19. Kutateladze, S.S. Convex operators. Russ. Math. Surv. 1979, 34, 181-214. [CrossRef]

20. Niculescu, C.P.; Olteanu, O. From the Hahn-Banach extension theorem to the isotonicity of convex functions and the majorization theory. Rev. R. Acad. Cienc. Exactas Fis. Nat. RACSAM 2020, 114, 171. [CrossRef]

21. Păltineanu, G.; Bucur, I. Some density theorems in the set of continuous functions with values in the unit interval. Mediterr. J. Math. 2017, 14, 44. [CrossRef]

22. Lemnete, L. An operator-valued moment problem. Proc. Am. Math. Soc. 1991, 112, 1023-1028. [CrossRef]

23. Bennett, G. Hausdorff means and moment sequences. Positivity 2011, 15, 17-48. [CrossRef]

24. Putinar, M. Positive polynomials on compact semi-algebraic sets. Indiana Univ. Math. J. 1993, 42, 969-984. [CrossRef]

25. Sokal, A.D. The Euler and Springer numbers as moment sequences. Expo. Math. 2020, 38, 1-26. [CrossRef]

26. Stoyanov, J.M.; Lin, G.D.; Kopanov, P. New checkable conditions for moment determinacy of probability distributions. SIAM Theory Probab. Appl. 2020, 65, 497-509. [CrossRef]

27. Vasilescu, F.H. Spectral measures and moment problems. In Spectral Analysis and its Applications (Ion Colojoară Anniversary Volume); Theta: Bucharest, Romania, 2003; pp. 173-215.

28. Norris, D.T. Optimal Solutions to the L $\infty$ Moment Problem with Lattice Bounds. Ph.D. Thesis, The University of Colorado, Boulder, CO, USA, 2002. Available online: http://math.colorado.edu/\$ $\backslash$ sim\$norrisdt/dougthesis.ps (accessed on 22 April 2021). 
29. Ambrozie, C.; Olteanu, O. A sandwich theorem, the moment problem, finite-simplicial sets and some inequalities. Rev. Roum. Math. Pures Appl. 2004, 49, 189-210.

30. Gosse, L.; Runborg, O. Resolution of the finite Markov moment problem. Comptes Rendus Math. 2005, 341, 775-780. [CrossRef]

31. Gosse, L.; Runborg, O. Existence, uniqueness, and a constructive solution algorithm for a class of finite Markov moment problems. SIAM J. Appl. Math. 2008, 68, 1618-1640. [CrossRef]

32. Lemnete-Ninulescu, L.; Zlătescu, A. Some new aspects of the L-moment problem. Rev. Roum. Math. Pures Appl. 2010, 55, 197-204.

33. Mihăilă, J.M.; Olteanu, O.; Udrişte, C. Markov-type moment problems for arbitrary compact and for some non-compact Borel subsets of $\mathbb{R}^{n}$. Rev. Roum. Math. Pures Appl. 2007, 52, 655-664.

34. Olteanu, $\mathrm{O}$. Application de théorèmes de prolongement d'opérateurs linéaires au problème des moments e à une generalization d'un théorème de Mazur-Orlicz, (Applications of theorems on extension of linear operators to the moment problem and to a generalization of Mazur-Orlicz theorem). Comptes Rendus Acad. Sci. Paris 1991, 313, 739-742.

35. Olteanu, O. New results on Markov moment problem. Int. J. Anal. 2013, 901318. [CrossRef]

36. Olteanu, O. Approximation and Markov moment problem on concrete spaces. Rend. Circ. Mat. Palermo 2014, 63, 161-172. [CrossRef]

37. Olteanu, O. Polynomial approximation on unbounded subsets, Markov moment problem and other applications. Mathematics 2020, 8, 165. [CrossRef]

38. Olteanu, O. On truncated and full classical Markov moment problems. Contrib. Math. 2021, 3, 29-36. [CrossRef] 